\title{
MAGNETIZATION RELAXATION IN CdMnS
}

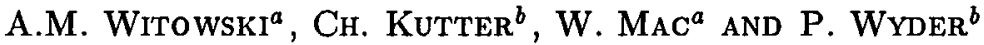 \\ ${ }^{a}$ Institute of Experimental Physics, Warsaw University \\ Hoża 69, 00-681 Warszawa, Poland \\ ${ }^{b}$ Hochfeld Magnetlabor Grenoble, MPI, 38042 Grenoble Cedex, France
}

\begin{abstract}
The static magnetization measurements and relaxation of magnetization data for two $\mathrm{Cd}_{1-x} \mathrm{Mn}_{x} \mathrm{~S}$ samples with different composition are presented. The magnetization is described by the modified Brillouin function. The magnetization relaxation shows unusual composition dependence. This behavior is explained by cross-relaxation to the unknown fast relaxing centers introduced during growth.
\end{abstract}

PACS numbers: $76.30 . \mathrm{Fc}$

Mn atoms introduced into II-VI crystals become $\mathrm{Mn}^{2+}$ ions creating ${ }^{6} S$ ground state. For low $\mathrm{Mn}$ compositions the materials are paramagnets with magnetization given by $\mathrm{Mn}$ spin alignment (e.g. [1]). Previously, we investigated the magnetization relaxation in CdMnTe [2] and CdMnSe [3]. We observed that the relaxation in CdSe based materials is slower than in CdMnTe with the same Mn composition as it has been observed in microwave EPR experiments [4]. Additionally, only very weak dependence on crystallographic orientation was observed. To extend our knowledge about the effect of anion type and crystallographic structure on spin dynamics we perform the measurements of magnetization relaxation in $\mathrm{Cd}_{1-x} \mathrm{Mn}_{x} \mathrm{~S}$. The mixed crystals based on CdS have wurtzite structure with local Mn environment of $C_{3 v}$ symmetry (in CdSe the local symmetry is almost $T_{d}$ ).

The used crystals were grown using a Bridgman method with nominal Mn concentration of $0.25 \%$ and $1.0 \%$. The composition was checked using energy dispersive X-ray fluorescence (EDXRF) and established to be $0.8 \%$ and $1.2 \%$ for our two samples. The microprobe analysis done on another part of the crystal from which the second sample was cut gives $x=1.2 \%$ with perfect agreements with our findings [5]. To check the static magnetic properties of both samples the magnetization was measured at $6 \mathrm{~K}$ up to $5 \mathrm{~T}$ using SQUID magnetometer. The results are depicted in Fig. 1. To these data the modified Brillouin function was fitted in the form $[6,7]$ :

$$
M(B)=M_{\mathbf{s}} B_{5 / 2}(y),
$$

where $B_{5 / 2}(y)$ is the Brillouin function with $y=5 \mu_{\mathrm{B}} B / k\left(T+T_{0}\right), M_{\mathrm{s}}$ is a technical saturation value (less than the true saturation $\times M_{0}$ ) and $T_{0}$ is a fenomenological parameter describing interaction between spins. $M_{\mathrm{s}}$ and $T_{0}$ are treated as fitting parameters and their obtained values are listed below:

$$
\begin{array}{llll}
x=0.8 \%, & M_{\mathrm{s}}=1.53 \mathrm{emu} / \mathrm{g}, & T_{0}=0.24 \mathrm{~K}, & M_{\mathrm{s}} / M_{0}=0.0078, \\
x=1.2 \%, & M_{\mathrm{s}}=2.59 \mathrm{emu} / \mathrm{g}, & T_{0}=1.98 \mathrm{~K}, & M_{\mathrm{s}} / M_{0}=0.0133 .
\end{array}
$$




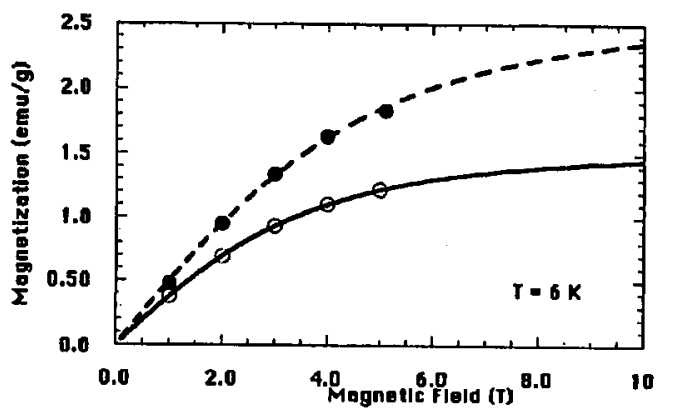

Fig. 1. The magnetization of two $\mathrm{Cd}_{1-x} \mathrm{Mn}_{x} \mathrm{~S}$ samples measured at $6 \mathrm{~K}$. The open and close circles describe experimental data for a sample with $x=0.8 \%$ and for a sample with $x=1.2 \%$, respectively. The solid lines represent fitted dependence described by Eq. (1) with the parameters listed in the text.

The value $M_{\mathrm{s}} / M_{0}$ is effective $\bar{x}$-value usually a little bit smaller than the real composition. In our case we found for our sample $\bar{x}$ to be very close to the $x$ values obtained with other methods. Both $\bar{x}$ values are not smaller than $x$, but in our experimental conditions $(T=6 \mathrm{~K}$ and $B \leq 5 \mathrm{~T})$ the measured magnetization is far from its saturation value and therefore the fit parameters are established with quite large errors. Nevertheless, the presented results suggest that the distribution of $\mathrm{Mn}$ ions in the crystals is almost random distribution.

The magnetization relaxation was measured using a "heat pulse" method described elsewhere [2]. The short laser pulses (tens and hundreds ns pulse duration) warm the lattice disturbing thermal equilibrium between lattice and $\mathrm{Mn}$ spin system. Changes of magnetization $M(t)$ relaxing toward its new thermal equilibrium induced voltage in the pick-up coil. After a short transition time the voltage decays exponentially suggesting the same time dependence of magnetization. The relaxation rates were established from the fit of exponential function to these data. The measurements were done at $4.5 \mathrm{~K}$ and in magnetic field up to $8 \mathrm{~T}$.

The obtained results are presented in Fig. 2. As can be seen for the sample with higher composition, at fields above $0.5 \mathrm{~T}$ magnetization relaxation is much slower (even an order of magnitude at fields higher than $3 \mathrm{~T}$ ) than in the sample with $x=0.8 \%$. In previous experiments the inverse dependence on composition has been observed $[2,3]$.

Such behavior was easy to understand. With increasing composition the average distance between spins decreases and interaction between spins becomes stronger (dipole type and/or exchange). Phonon modulation of the interaction is responsible for the spin-lattice relaxation. Therefore, the presented unusual dependence of relaxation rates on composition cannot be explained considering interaction between spins, only. During slightly different growth process in two samples a different concentration of unknown fast relaxing centers is introduced. Thus, the relaxation process probably is going in two steps. First, the spin polarization is transferred from manganese ions to the unknown centers (like defects or 


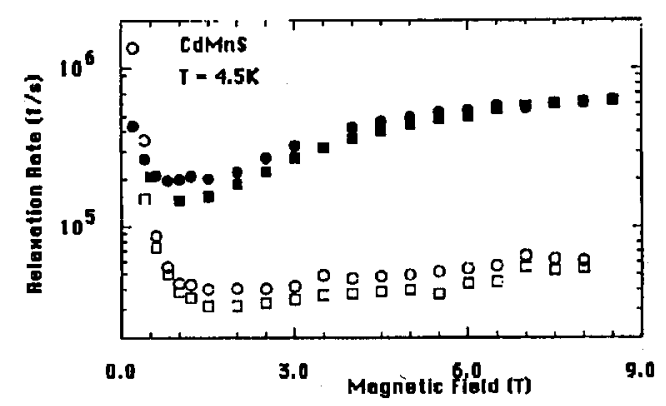

Fig. 2. The relaxation rate for two $\mathrm{Cd}_{1-x} \mathrm{Mn}_{x} \mathrm{~S}$ samples measured at about $4.5 \mathrm{~K}$. The circles denote data taken for $c$ axis parallel to the external magnetic field, and the squares represent data for $c$ perpendicular to the field. The closed and open symbols are for samples with $x=0.8 \%$ and $x=1.2 \%$, respectively.

impurities) by the cross-relaxation. Then, in these centers the spin flip occurs by the spin-lattice relaxation process. The presented model is additionally supported by the magnetic field dependence of relaxation rates.

The data shown in Fig. 2 could not be explained by existing models of spin-lattice relaxation of isolated spins. Because the presented results are strongly dependent on taken samples, they cannot be compared with the previous results on CdMnTe and CdMnSe. Also the dependence on crystallographic orientation cannot be related to the behavior of spin-lattice relaxation of isolated manganese spins.

\section{Acknowledgments}

The authors would like to acknowledge the EDXRF measurements performed by Dr. W. Dobrowolski. Also we would like to thank Dr. hab. A. Twardowski for the help during magnetization measurements. This work has been partially supported by the Committee for Scientific Research through grant \# 224109203.

\section{References}

[1] J.K. Furdyna, J. Appl. Phys. 64, R29 (1988); W.J.M. de Jonge, H.J.M. Swagten, J. Magn. Magn. Mater. 100, 322 (1991).

[2] T. Strutz, A.M. Witowski, P. Wyder, Phys. Rev. Lett. 68, 3912 (1992); T. Strutz, A.M. Witowski, P. Wyder, Rev. Sci. Instrum. 64, 1853 (1993).

[3] T. Strutz, A.M. Witowski, P. Wyder, to be published.

[4] R. Kremer, J.K. Furdyna, Phys. Rev. B 31, 1 (1985).

[5] M. Nawrocki, private communication.

[6] J. Gaj, R. Planel, G. Fishman, Solid State Commun. 29, 435 (1979).

[7] D. Heiman, Y. Shapira, S. Foner, Solid State Commun. 45, 899 (1983). 